\title{
Controlled Reactivation of Psycho-Semantic Space by Subconscious (Subthreshold) Stimuli
}

\author{
Semyon Ioffe ${ }^{1}$, Sergey Yesin ${ }^{2}$ \\ ${ }^{1}$ Department of the Psycho-semantics, Northam Psychotechnologies, Ottawa, Canada; \\ ${ }^{2}$ Psychophysiology Lab, Vishnevsky Central Military Clinical Hospital, Krasnogorsk, Russia. \\ Email: sioffe@primus.ca \\ Received December 24 $4^{\text {th }}, 2010$; revised February $24^{\text {th }}, 2011$; accepted March $29^{\text {th }}, 2011$.
}

\begin{abstract}
Psycho-Semantic spaces were constructed for 25 volunteers ( 15 smokers and 10 non smokers) BEFORE and AFTER their exposure to the "defence reaction" development procedure to the word COBRA and subconscious teaching. Subconscious teaching was done to disassociate each participant's psychological interrelationship towards smoking - cigarette brands. We have established that defence reaction corresponds with the basic emotion of FEAR. One can evaluate the degree of forming a defence reaction in the subject being tested from reduction of distance from the word COBRA to the emotion FEAR and defence reaction has a moderate relationship with the development procedure (number of errors - number of reinforcements). We have also shown the transfer of the defence reaction from the word COBRA to the words shown in the subconscious mode before the word COBRA $(p<0.05)$ and changes of the emotional relationship to cigarette brands using the anchoring technique - subconscious semantic teaching verbiage type BOND-FEAR and others $(\mathrm{p}<0.05)$. We learned that PsyExpert and SMART technologies are effective and sensitive methodologies for studying the psycho-semantic space of emotions, control of reactivation of individual psycho-semantic space and effectively retraining the subconscious mind.
\end{abstract}

Keywords: Unconscious Mind, Reactivation, Psycho-Semantics, Subconscious Re-Teaching, Defence Reaction, Smoking

\section{Introduction}

The name Psycho-semantics derives from two words: PSYCHE (the mind) and SEMANTICS (the study of meaning). Many researchers have allocated a special role to semantic factors (i.e., semantic, information) during the human life (Putnam, 1975; Peacocke, 1992; Smirnov, Beznosjuk, \& Zhuralyov, 1995).

In the process of the evolution of the psyche, the role of semantic signals has grown. A reaction to a particular stimulus results from activation of memory contents that corresponds to the stimulus in question.

Any perceived information immediately causes an associative chain of corresponding memories. This suggests that any perceived stimulus is not stored in memory in the form of an independent semantic element but stored only as an aggregate of associative connections with other elements (Meyer, 1970; Smith, Shoben, \& Rips, 1974; Smirnov et al., 1995).

Any internal or environmental change entails change of mental activity and a respective alteration of behavior. If this stimulus is accompanied with significant life changes, a special meaning is appropriated to it. The meaning of stimuli (which the word or an image represents) is its connection and association with concrete changes of the emotional state. Thus, having determined a group of significant words that are emotionally associated for the individual, it becomes possible to change the person's mental activity and behavior in a different way by showing these words to the participant (Smirnov et al., 1995; Ioffe, Yesin, Afanasjev, \& Nezhdanov, 2007a, b).
A measure of the importance of a word is the quantity of the associative connections to other words. On the basis of such words, a base semantic nucleus of personality is created (Smirnov et al., 1995; Schreiber \& Nelson, 1998; Ioffe \& Konobeevsky, 2008) allowing understanding of the principles of reaction of the person's psyche to different stimuli. Thus, drawing up a base semantic nucleus of personality, for example, as a psychological portrait of the person being tested, allows researchers to determine the subject's true attitude to different spheres of life and activity; to answer the most innate questions; and to reveal motivation and propensities to addictive behavior, dependencies, etc. (Smirnov et al., 1995).

Controlled subconscious reactivation and teaching represents a process of activating the traces of semantically meaningful issues symbolized by words and emotions - associate or disassociate - and repositioning them into a different psycho-semantic space, creating stable beneficial meanings to the human personality.

Semantic space in humans and their dynamics can be investigated using various well established rigorous techniques: semantic differential (Osgood, Succi, \& Tannenbaum, 1957; Osgood, 1959, 1967, 1976) personal construct theory (Kelly, 1970), classification of the emotions theory (Leontiev, 2002), Max Luscher's color personality test. Using them one by one, comparing results and drawing combine conclusions requires significant time of the subject being tested and is extremely labour intensive for the researcher.

A computer administrated PsyExpert test (Ioffe, \& Yesin, 2010) which combines in one test procedure all the above de- 
scribed rigorous techniques was used in our study. PsyExpert allows visualize dynamic changes of semantic space in the form of Runge sphere using emotions as coordinates. The very heart of PsyExpert is a concept of innate relations of human emotions and feelings with color. PsyExpert uses classification of emotions by Leontiev which summarizes work by Ekman (1999), Ekman and Davidson (1994); Izard (1977) and others and suggests a universal classification of the emotions (Leontiev, 2002). The corner stone of this classification are eight basic emotions representing human personal needs. (http://emoatlas.narod.ru/book1.html) The PsyExpert test incorporates an equal number of basic emotions, connected with individual needs of the personality (positive and negative, directed on itself or on the object, anticipating or ascertaining).

The second complex successively used in a number of publications (Ioffe, Yesin, Afanasjev, \& Nezhdanov, 2007a, 2007b; Ioffe \& Konobeevsky, 2008; Ioffe \& Yesin, 2010) is nonverbal assessment and teaching of the subconscious mind-SMART (Semantic Mediated Analysis of Responses and Teaching). Nonverbal examination bypasses the conscious "censorships" and consists of registration of non realized reactions of the subject being tested, as an "answer" to a presentation of the semantic stimulus in the subconscious "invisible" subthreshold range. The subthreshold range is positioned between the physiological threshold and comprehension threshold and stimuli shown in this range has short presentation time that registers through the retina into the brain "without comprehension." The modified complex of the visual-motor act is used as a response of the non realised reaction. Based on the character of the changes of these reactions the conclusion of their importance is drawn for each semantic stimulus or group of stimuli in psycho-semantic space of the subject. And as both "question" and "answer" bypasses the conscious mind, the examination is able to reveal true motivation, aspirations, system of vital values, destructive tendencies, participation in illegal actions, affects, mental and psychological problems and their reasons, various kinds of dependences (addiction to alcohol, narcotics, toxic substances, foods, gambling, etc.) and many other issues (Smirnov, Beznosjuk, \& Zhuravlyov, 1995; Ioffe, Yesin, Afanasjev, \& Nezhdanov, 2007; Ioffe \& Konobeevsky, 2008).

Another extremely important feature of SMART is the technique has the module which allows subconsciously reactivate psycho-semantic fields and re-teaches the subconscious mind. In this technology teaching verbiage is shown in a subconscious mode and directs desirable changes of the patient for the future (Ioffe, Yesin, Afanasjev, \& Nezhdanov, 2007b).

To learn dynamics of conscious and subconscious influences on the subconscious mind we have decided to investigate how easy a subject's semantic space can be influenced by simple control situations:

1) Classical conditioning associated with the defence reaction and its transformation into conditional defence response in the subconscious mind;

2) Usage of the newly developed conscious and subconscious conditional stimuli in the procedure of subconscious re-teaching;

3) Comparing the influence of subconscious conditional stimuli with other verbiage used in re-teaching.

More specifically the following objectives were the goals of our study:

1) To evaluate the dynamic changes of the patient's psycho- semantic space to the influences of conscious and subconscious stimuli.

2) To define emotions corresponding to "defence reaction" of the subject and develop "defence reaction" to the word COBRA (any other word can be used for this purpose, but we have chosen to use the word COBRA) using a standard conscious methodology approach.

3) To investigate the transfer of the "defence reaction" to the words-stimulus shown subconsciously before the word COBRA.

4) To quantify the changes of psycho-semantic space during the subconscious presentation of the teaching verbiage.

\section{Method}

\section{Participants}

Twenty five subjects, healthy volunteers, all men ages $20.8 \pm$ 1.9 years participated in the study. Out of 25 men studied, 15 were smokers for $4.3 \pm 2.9$ years and smoked $12.1 \pm 7.0$ cigarettes per day. Each participant signed a consent form. To achieve a uniformity of the study sample for psychological health each participant was subjected to the MMPI test. All participants had shown no obvious clinical pathology and were recognised as a homogeneous research group suitable for carrying out further study.

\section{Procedure}

Each participant was subjected to the following regiment:

1) Each participant was asked to write 2 names of preferred (for smokers) or well-known to them (for non-smoking) brands of cigarettes. Further in the text above first brands on the list were named accordingly as BRAND CIGARET1 and second brand on the list BRAND_CIGARET2. These topic names were used in PSYEXPERT and SMART testings.

2) The psycho-semantic space for each participant was constructed, all objects of study were defined and precisely positioned, distances between the basic emotions of the participant and elements were calculated: word COBRA, BRAND of CIGARETTES (its own brand for each participant).

3) Each participant was exposed to conscious and subconscious stimuli for 4 to 5 minutes at approximately one stimulus per second. The participant was asked to press the mouse button at the appearance of any words on a screen monitor, except for the word COBRA (conscious presentation). When the participant made a mistake, a strong auditory message (100 decibels) through headphones, about the error, was delivered, which the participant tried to avoid since the "defence reaction" to this word was developed. This was tested by subconscious presentation of the word COBRA to understand if conscious procedure of development of the defence reaction was transferred to the subconscious mind. The number of errors during development of the defence reaction was calculated for further analysis. Concurrently subconscious stimuli were delivered to the subject being tested in two variants:

a) Before a visible word COBRA appeared "invisible" BRAND_CIGARET1 in the mode of "perceptions without comprehension" subconsciously.

b) During the teaching process semantic teaching verbiage types of BRAND_CIGARET2-FEAR were shown subcon- 
sciously.

4) Each participant's psycho-semantic space was reconstructed after the subconscious teaching procedure.

Reliability of the PsyExpert results was investigated using a degree of conformity of the word COBRA with colour. In the course of testing it was required to correlate the word COBRA with colours of a spectrum and to evaluate the strength of the association from 1 to 10 (10 has been accepted for $100 \%)$. If the average conformity would turn out to be much below $50 \%$ we could not depend reliably on the test results (Ivanter, 1992).

The PsyExpert test comprises of a number of steps:

1) The subject being tested looks through a number of pictures illustrating various life situations, recognizes some as opposite pairs of emotions, names them and undersigns. It is natural that the emotions especially relevant to the subject being tested are "recognized" with ease.

2) The colors of a visible spectrum are shown one by one and the subject being tested is asked to select for each of the emotions (poles of the constructs) revealed in the previous step, the most "desirable" colors and evaluate their strength using a scale from 1 to 10 .

Consequently, pairs of opposite emotions (the constructs) are replaced with corresponding pairs of colors.

3) Evaluation of the elements with these color associative pairs is carried out.

Evaluating the elements by colors, the subject being tested does not suspect that he/she is actually "associating" each element with an individual specific emotion (the pole of the construct), thus openly revealing their inner world.

4) In conclusion, the colors are ranked by the subject being tested in the order of desirable-undesirable colors. Identified emotions (poles of constructs) and elements are all included in the same order of colors.

Finally, calculation is provided for a number of statistical indices: color correlation, index of cognitive differentiation (connection intensity), exact distances between all the objects in psycho-semantic space and conflicts of personality of self identification.

The test results are displayed in graphic form as a 3D color psycho-semantic space represented as a Runge's Sphere with basic emotions, constructs and elements situated within and also displayed in a printable form comprising a detailed description of analysed psycho-semantic space.

SMART technology is based on the universal principles of human behaviour and scientific experimentation: Humans are the product of information of their surrounding environment. Any traits, influences, abilities, etc. are described and experienced through words, pictures, sound etc. Information is categorized and prioritized by emotions throughout one's life. The major content of the human's informational being is not accessible to his/her conscious mind. It belongs to the subconscious mind. SMART technology uses the universal principles of scientific experimentation: each experiment consists of: Control Probe/Stimuli, Reper (Reference point) and a Registered response (Smirnov, Beznosjuk, \& Zhuravlyov, 1995; Ioffe, Yesin, Afanasjev, \& Nezhdanov, 2007). Each person is his own control, since every person's psyche is different. It is important to understand that every individual test is a complete scientific experiment since it contains all the above-mentioned components. Controls represent stimuli which have no meaning to the subject. They are in the form of a row of randomly chosen 15 numbers that flash across the screen at a fraction of a second, registering through the retina into the brain. This control is then masked by a different row of randomly-chosen 15 numbers. The first row, the control, is seen subconsciously. The second row, the masker, is seen consciously. The probe is semantically meaningful stimuli in the form of a word that moves across the screen at a fraction of a second, registering through the retina into the brain. This probe/word is then masked by a row of randomly-chosen 15 numbers. The probe/word is seen subconsciously and the masker row of numbers is seen consciously. Reper is a different kind of control. It is a measurement developed to gauge defence reaction (the subject's reaction to the "punishment" they receive during the test). This reaction is then measured to understand how the subject's subconscious mind responds defensively. Registered Response is when the subject is asked to press a mouse button each time they see a row of numbers. The combined visual and motor reaction to the controls, probe and reper are measured by the response time between a stimuli and the subject pressing the mouse button. Rigid requirements are placed on the respondent ensuring that they are unable to alter the test results in any way. The speed at which they react is measured using statistical analysis. The relationship between the control, probe and reper is analyzed by measuring deviations between the three components.

\section{Statistics}

Statistical calculations were carried out using nonparametric criteria of Lehmann-Rosenblatt for homogeneity test (Lehmann, 1951; Rosenblatt, 1952), Pearson's correlation coefficient sensitive to a linear relationship between two variables and Spearman's rank correlation coefficient (nonlinear correlation) $r_{s}$ as a non-parametric measure of statistical dependence between two variables. Statistical significances of the coefficients were calculated. Significance level at $95 \%(\mathrm{p}<0.05)$ was accepted. In addition, we used general classification of association of correlation according to Ivanter, 1992. The association was considered to be strong if the correlation factor $r>0.70$; average if $0.50<r<0.69$; moderate if $0.30<r<0.49$; weak if $0.20<r<$ 0.29 ; and very weak if $r<0.19$.

\section{Results}

\section{The Psycho-Semantic Space Dynamics for the Emotions and "Defence Reaction", and Development of "Defence Reaction" to the Word COBRA}

When we tested all participants BEFORE the defence reaction development procedure to the word COBRA, we observed in the psycho-semantic space the closest negative emotion to the word COBRA (Table 1) emotion of FEAR (25.0) which was "inside" of one sigma range (26.8). This fact reflects the initial psychological state of the participants which is in full conformity with Leontief's theory of emotions: "cobra" is a snake, can bite, it is possible to die-anticipating emotion (though yet have not bitten, but can bite!) and directed on itself (to bite not someone, but!). "Fear", according to Leontief is also the anticipating emotion directed on the self. AFTER defence reaction development procedure to the word COBRA, we see in the psycho-semantic space the closest negative emotion to the word COBRA is also the emotion FEAR (Table 1). The 
Table 1 .

Dynamics of changes of average psycho-semantic distances from the word COBRA to negative emotions BEFORE defence reaction development procedure to the word COBRA $(N=25$, Average Sigma $=26.8)$ and AFTER defence reaction development procedure to the word COBRA $(N=25$, average Sigma $=27.9)$. All distances in the table represented by Mean $\pm S D$.

\begin{tabular}{lcccc}
\hline & Neg. emotions & Anger & Grief & Fear \\
\hline BEFORE & & $27.0 \pm 16.1$ & $29.6 \pm 18.7$ & $25.0 \pm 15.2$ \\
AFTER & $25.5 \pm 15.4$ & $26.3 \pm 16.3$ & $19.8 \pm 8.0$ \\
Lehmann-Rosenblatt $\mathrm{T}=0.46$ & 0.17 & 0.23 & $38.8 \pm 24.3$ & $0.51^{*}$ \\
\hline
\end{tabular}

$* \mathrm{p}<0.05$

Table 2 .

Results of the statistical analysis of 25 participants.

\begin{tabular}{|c|c|c|}
\hline \multirow{2}{*}{ Relation between decrease of the COBRA-FEAR distances and number of errors. } & Pearson correlation $\mathrm{R}$ & Spearmen correlation Rs \\
\hline & -0.36 & $-0.46^{*}$ \\
\hline
\end{tabular}

$* \mathrm{p}<0.05$

Table 3.

Results of statistical analysis of the distances for words COBRA and BRAND_CIGARET1, negative emotion FEAR and BRAND_CIGARET2 and a positive emotion HOPE and BRAND_CIGARET2 for 25 participants BEFORE and AFTER anchoring procedures.

\begin{tabular}{ccc}
\hline A & BEFORE superimposition procedure & AFTER superimposition procedure \\
COBRA-BRAND_CIGARET1 & $39.3 \pm 17.9$ & $30.9 \pm 16.7$ \\
B & BEFORE anchoring procedure & AFTER anchoring procedure \\
FEAR-BRAND_CIGARET2 & $38.5+17.4$ & $34.3+16.5$ \\
HOPE-BRAND_CIGARET2 & $43.3 \pm 26.3$ & $\mathrm{p}<0.1$ \\
$\mathrm{p}<0.05$ & $54.0 \pm 28.7$ \\
\hline
\end{tabular}

distance after the procedure has decreased, which means these two concepts became more subconsciously associated than before the procedure. We calculated statistical differences using criteria of Lehman-Rosenblatt for distances from the word COBRA to negative emotions BEFORE and AFTER defence reaction development procedure to the word COBRA (Table 1). Only dynamics of the emotion FEAR have shown statistically significant changes to defence reaction development procedure using the word COBRA $(p<0.05)$. For all of the participants a summary data were compiled for distances between the word COBRA and the FEAR emotion BEFORE and AFTER defence reaction development procedure to the word COBRA. Their differences and the number of errors made by each participant and dependency of the number of errors made by participants during testing on the defence reaction development to the word COBRA was evaluated (Table 2) using linear and nonlinear correlation. Though it seems that a larger number of errors correspond with larger differences which suggest the word COBRA is closer to the emotion of Fear in psycho-semantic space, the statistically significant correlation shows only a moderate relationship between the number of errors and closeness of the COBRA-FEAR position in psycho-semantic space according to classification by Ivanter 1992. In addition, we can suggest that a nonlinear relationship between forming a defence reaction and the number of errors was more sensitive than the linear.

\section{Features of Appearance of "Defence Reaction" to the Words-Stimulus Shown in the Subconscious Range}

We have studied how the developing defence reaction to the word COBRA in the subject being tested transferred to the words-concepts shown in the subconscious range before the word COBRA.

In the present part of the study, participants were exposed subconsciously to one of the cigarettes brand names before the word COBRA (BRAND_CIGARET1, Marlboro, Winston etc.). We observed statistically significant $(p<0.05)$ changes of mentality in the form of reduction of average psycho-semantic distance after the procedure of subconsciously showing a cigarettes brand name before the word COBRA, meaning the tendency of the emotional equation of the reaction of the participant to the word COBRA and a word, representing the brand of cigarettes (Table $3(\mathrm{~A})$ ).

\section{Efficiency of Anchoring-Presentations in Subconscious Mode of the Teaching Verbiage Consisting of Positive and Negative Components, for Example FEAR-BOND ("Bond"-Cigarettes Brand)}

We have observed the changes of mentality in the form of reduction of average psycho-semantic distance after the anchoring procedure, meaning there was a tendency of the emotional equation of the reaction of the participant to the word FEAR and a word, representing a brand of cigarettes (Table 3 (B)), but it was only approaching statistical significance $(\mathrm{T}=$ $0.39, \mathrm{p}<0.1)$. As we have not obtained statistically significant changes in analysis of the group which included both smoking and non smoking participants, we decided to analyze the data separately for smoking and non smoking subjects. Results of this approach are presented in Table 4. As we can see from the data (Table 4) the teaching procedure of anchoring is more 
effective in the non smoking group $(\mathrm{p}<0.05)$ rather than in smoking subjects. This might occur because the intrinsic readiness to feel negative emotions to cigarettes by non smokers is higher than smokers. We have chosen another approach to investigate an anchoring procedure (FEAR-BRAND_CIGARET2) on the dynamics of the positive emotion HOPE (opposite and complimentary to the emotion "Fear") and BRAND_CIGARET2 provided data in Table 3 (B). We observed a change of mentality in the form of an increase in average psychosemantic distance after the anchoring procedure (FEAR-BRAND CIGARET2) which symbolizes the tendency of emotional separation of the positive emotion HOPE and a word representing a cigarette brand.

\section{Validity of the Results}

We have observed a high degree of conformity of the positive and negative emotions with colour corresponding shades of a colour palette presented in the test in all our participants, which on average was from $(95.6 \pm 10.6) \%$ and $(90.9 \pm 16.0) \%$ (Table 5). The calculations of the average value of the association of the word COBRA with colors were $(77.2 \pm 21.5) \%$ BEFORE and $(77.6 \pm 22.5) \%$ AFTER exposure to the SMART testing/teaching procedure provides statistically insignificant results. For all other words-concepts (elements) 2 tests of association with colors were done for each participant: BEFORE and AFTER exposure to SMART testing/teaching procedures (Table 6). The calculations of the average value of the association of the elements RAND_CIGARRET1 and BRAND_ CIGARRET2 with colors were accordingly BEFORE $83.3 \pm$ 21.9 and $76.7 \pm 24.0$ and AFTER $82.9 \pm 19.6$ and $74.8 \pm 27.5$. Both sets of results showed no statistical significance $(\mathrm{p}>0.1)$.

\section{Discussion}

We have provided evidence that both procedures of subconscious teaching superimposed and anchoring verbiage can be used to change the mentality of the subject. Defence reaction corresponds with the basic emotion FEAR, the anticipating negative emotion directed on self (Leontief, 2002). By the reduction of distance from the word COBRA to the emotion FEAR one can evaluate the degree of forming a defence reaction in the subject being tested. Defence reaction has a moderate relationship with the number of errors (number of reinforcements). We have shown that PSYEXPERT technology is a sensitive methodology in the study of psycho-semantic space of emotions and follows up the changes of mentality after psychotherapeutic manipulations. The necessity to check the validity of the PSYEXPERT results arises from the method which works on the basis of colour associations. Therefore accuracy of all results of the test depends on the accuracy of the selection of shades of a colour palette to words-concepts by the subject being tested. We have investigated the degree of conformity of the positive and negative emotions with colour corresponding shades of a colour palette presented in the test to all our participants. The associations of negative and positive emotions with colour have shown a high degree of confidence of correlation of corresponding emotions and colours in all participants, i.e. high validity of the results of the present study. For the words-concepts the tests of association with colors BEFORE and AFTER exposure to SMART testing or teaching procedures did not change the association between the word COBRA and color. According to our calculations, the average value of conformity of the word COBRA with colour was $(77.2+$ $77.6) / 2=77.4 \%$. It testifies that participants could cope very

Table 4.

Average distances between emotion FEAR and BRAND_CIGARET2 for smoking and non smoking participants BEFORE and AFTER anchoring procedures.

\begin{tabular}{lccc}
\hline & BEFORE anchoring procedure & AFTER anchoring procedure & P \\
\cline { 2 - 4 } Smokers $\mathrm{N}=15$ & $40.1+19.2$ & $39.6+18.3$ & $\mathrm{p}>0.1$ \\
Non smokers $\mathrm{N}=10$ & $36.9+15.6$ & $29.0+14.7$ & $\mathrm{p}<0.05$ \\
\hline
\end{tabular}

Table 5 .

Association of positive and negative emotions with color (\%).

\begin{tabular}{ccccc}
\hline Positive emotion & HOPE & PLEASURE & SATISFUCTION & INTEREST \\
\hline $\mathrm{M} \pm \mathrm{SD}$ & $91.2 \pm 17.3$ & $92.4 \pm 13.6$ & $90.4 \pm 16.6$ & $89.5 \pm 16.4$ \\
Negative emotion & FEAR & GRIEF & ANGER & NEGLECT \\
$\mathrm{M} \pm \mathrm{SD}$ & $96.0 \pm 9.4$ & $92.8 \pm 15.1$ & $95.6 \pm 11$ & $97.9 \pm 6.9$ \\
\hline
\end{tabular}

Table 6.

Association of the elements with color BEFORE and AFTER SMART testing/teaching procedures (\%).

\begin{tabular}{cccc}
\hline & COBRA & BRAND_CIGARRET1 & BRAND_CIGARRET2 \\
\hline BEFORE & $77.2 \pm 21.5$ & $83.3 \pm 21.9$ & $76.7 \pm 24.0$ \\
AFTER & $77.6 \pm 22.5$ & $82.9 \pm 19.6$ & $74.8 \pm 27.5$ \\
$\mathrm{M} \pm$ SD & $77.4 \pm 22.0$ & $83.1 \pm 20.7$ & $75.8 \pm 25.8$ \\
$\mathrm{P}$ & $\mathrm{p}=0.1$ & $\mathrm{p}>0.1$ & $\mathrm{p}>0.1$ \\
\hline
\end{tabular}


well with the tasks and were confidently associating the word COBRA with colour well above $70 \%$. Hence, we can use the results of such association of the words and colours with full confidence. The same can be said about other words-concepts (elements) participating in the test $(83.1 \pm 20.7$ and $75.8 \pm 25.8$ accordingly). The average for all elements in tables 5 and 6 was $(88.4 \pm 16.5) \%$ which suggests high validity and accuracy of the test.

Resulting changes can be observed even after nonrecurring five minutes of subconscious teaching superimposed and anchoring verbiage. Ioffe, Yesin, Afanasjev, Nezhdanov, (2007b) have shown that empirically applied procedures of subconscious teaching 2 to 3 sessions per week for a total of 10 sessions, 60 to 90 minutes each, produced stable positive results in PTSD subjects with alcohol dependencies after 3 months. However outstanding questions still are: how long the psyche, based on observation of the psycho-semantic space remains retrained and what are the optimal conditions (how many sessions are required and what is the optimal length of a single session) for permanent changes of the psycho-semantic space human reactions? Further investigations are needed to provide answers to the above stated questions.

\section{Conclusion}

1) PSYEXPERT technology has been shown as an effective tool for evaluation and visualisation of the changes of the psycho-semantic space of the subject being tested to affects of various stimuli. When conscious stimuli COBRA were reinforced by "punishment" - a strong audio signal of error, we observed changes of the psycho-semantic space corresponding to development of a defensive reaction $(\mathrm{p}<0.05)$. Corresponding changes of the psycho-semantic space were observed to the effects of subconscious (subthreshold) stimuli and semantic teaching verbiage $(\mathrm{p}<0.05)$.

2) FEAR is an emotion which is emerging during development of the defence reaction in the subject being tested to the word COBRA $(\mathrm{p}<0.05)$.

3) We have proven the transfer of the defence reaction from the word COBRA to the words shown in the subconscious mode superimposed with the word COBRA $(\mathrm{p}<0.05)$.

4) We have shown change of the emotional relationship to cigarette brands using anchoring technique-subconscious semantic teaching verbiage type KENT-FEAR and others $(\mathrm{p}<$ $0.05)$.

5) Technologies used in our study open the door to controlled reactivation of individual psycho-semantic space and effective retraining of the subconscious mind.

\section{References}

Ekman, P. (1999). Basic emotion. In T. Dalgleish and M. Power (Eds.). Handbook of Cognition and Emotion. Sussex, U.K.: John Wiley \& Sons, Ltd.
Ekman, P., \& Davidson, R. J. (1994). The nature of emotion. Fundamental Questions. Oxford: Oxford University Press.

Ioffe, S., Yesin, S. V., Afanasjev, B. G., \& Nezhdanov I. K. (2007a). Psychosemantic diagnosis of alcoholic dependencies tested at the subconscious level in military personnel with posttraumatic stress disorder (PTSD). Polygraph Journal, 36, 57-69.

Ioffe S., Yesin, S. V., Afanasjev, B. G., \& Nezhdanov, I. K (2007b). The role of subconscious effects during the treatment of posttraumatic stress disorder with alcohol dependencies in military personnel. Polygraph Journal, 36, 133-148.

Ioffe, S., \& Konobeevsky, M. (2008). Psycho-semantic spheres of the personality among correctional facility employees. Journal of Police and Criminal Psychology, 23, 23-34. doi:10.1007/s11896-008-9018-7

Ioffe S., \& Yesin, S. (2010). Psyche From Within: Three Clinical Case Studies. Psychology, 1, 282-294. doi:10.4236/psych.2010.14037

Izard, C. E. (1977). Human Emotions. New York: Plenum Press.

Ivanter, E. V. (1992). Bases of Biometrology: Introduction in the Statistical Analysis of the Biological Phenomena and Processes. Petrozavodsk: Petrozavodsk Press, State Univesity.

Kelly, G. A. (1970). Behaviour is an Experiment. Perspectives in Personal Construct Theory. London: Academic Press.

Leontiev, V. O. (2002). Classification of the Emotions. Odessa: Innovation centre. http://emoatlas.narod.ru/book1.html

Lehmann, E. L. (1951). Consistency and unbiasedness of certain nonparametric tests. Annals of Mathematical Statistics, 22, 165-179.

Meyer, D. E. (1970). On the representation and retrieval of stored semantic information. Cognitive Psychology, 1, 242-299.

Osgood, C. E., Suci, G. J., \& Tannenbaum, P. H. (1957). The Measurement of Meaning. Urbana, IL: Illinois Press.

Osgood, C. E. (1959). Representational model and relevant research methods. In Pool I. (Ed.), Trends in Content Analysis. Urbana, IL: Illinois Press.

Osgood C. E. (1967). Semantic differential technique in the comparative study of cultures. L. A. Jakobovite and M. S. Miron (Eds), Readings in the Psychology of Language. Englewood Cliffs: Prentic-Hall.

Osgood, C. E. (1976). Focus of Meaning. Mouton, Hague.

Peacocke, C. (1992). A Theory of Concepts. Cambridge: MIT Press.

Putnam, H. (1975). The meaning of “meaning”. In K. Gunderson (Ed.), Language, Mind and Knowledge: Minnesota Studies in the Philosophy of Science. Minneapolis: University of Minnesota Press.

Rosenblatt, M. (1952). Limit theorems associated with variants of the von Mises statistic. Annals of Mathematical Statistics, 23, 617-623.

Schreiber, T. A., \& Nelson, D. L. (1998). The relation between feelings of knowing and the number of neighbouring concepts linked to the test cue. Memory \& Cognition, 26, 869-883.

Smirnov, I., Beznosjuk E., \& Zhuravlyov, А. (1995). Психотехнологии: компьютерный психо-семантический анализ и психокоррекция на неосознаваемом уровне. (Psychotechnologies: Computer psychosemantic analysis and psychocorrection at subconscious level). M. Culture.

Smith, E. E., Shoben, E. J., \& Rips, L. J. (1974). Structure and process in semantic memory: A featured model for semantic decisions. Psychological Review, 1, 214-241. 